\title{
Hydrogenic Impurity in Ridge Quantum Wire
}

\author{
E. Sadeghi and R. Khordad \\ Department of Physics, Yasouj University, Yasouj, Iran 75914-353
}

Received on 8 February, 2006

\begin{abstract}
The binding energies as well as wave functions of hydrogenic impurities located in V-groove $G a A s / A l_{x} G a_{1-x} A s$ quantum wires are calculated for different positions of the impurity inside the wires. The variational method is used and the carrier ground states are analytically calculated by an effective potential scheme together with a suitable coordinate transformation that allows the decoupling of the two-dimensional Schrodinger equation. The results are in good agreement with experimental points and other previous investigations.
\end{abstract}

Keywords: Hydrogenic impurity; Binding energy; V-groove quantum wire

\section{INTRODUCTION}

In the past few years, there has been an increasing interest in the electronical and optical properties of quantum wires. As the quantum confinement leads to the enhancement of the density of states at specific energies and increases the importance of excitonic effects by the modification of electron-hole Coulomb interaction [1], one-dimensional quantum wire structures have wide potential applications in future optical devices. Some novel techniques have been successful in obtaining a certain type of heterostructure known as ridge quantum wires. These ridge quantum wires have been obtained for a variety of materials such as InGaAs/GaAs [2], InGaAs/AlGaAs [3], InGaAs/InP [4] and GaAs/AlGaAs [5]. Due to the unusual form of the barrier profile of V-groove quantum wires, the calculation of hydrogenic impurity states may become impractical if the method of calculation of the carrier ground states is also numerically intensive. Several theoretical approaches have been put forth for the calculation of the energy levels and wave functions: Pescetelli et al [6] used a tight-binding method for $\mathrm{V}$-shaped quantum wire, Sa' ar et al [7] proposed a local-envelope function, and Ammann et al [8] used a quasi-factorization scheme. In the latter case, the wave functions were calculated for an adiabatic approximation of the potential profiles which therefore limits the calculation to smoothly angled, V-shaped wires. The binding energy of a hydrogenic impurity in a cylindrical quantum wire has been calculated as a function of location of the impurity with respect to the axis of the quantum wire in [9]. The effect of the shape of a cross-section on the impurity binding energies for a quantum wire has been investigated in [10]. However, in general the 2-dimensional Schrödinger equation in the effective mass approximation has been calculated numerically using either plane-wave expansion [11-13] or by adapting finite element methods [14]. In all cases, the methods employed require considerable numerical effort and impose major restrictions for their use in calculating electronic and optical properties.

Recently, a coordinate transformation method was used to study the electronic structures around corrugated interfaces and in $\mathrm{V}$-groove quantum wires. By this method, the nonplanar boundaries become a confining potential and the boundary conditions of electronic wave functions can be satisfied exactly on the interfaces after the coordinate transformation.

In this paper the method of effective potential [15] is used and the 2D Schrödinger equation is analytically solved [16]. Then, the variational method is applied and the binding energies of hydrogenic impurity are calculated. The method provides energy levels and wave functions that are in good agreement with more complicated calculations.

\section{THEORY}

In the effective mass approximation within envelope function formalism, the single particle Hamiltonian in V-grooved quantum wire can be written:

$$
H(\mathbf{r})= \begin{cases}\mathbf{P}^{2} /\left(2 m_{1}\right) & \mathrm{y}_{2}(\mathrm{x})<\mathrm{y}<\mathrm{y}_{1}(\mathrm{x}) \\ \mathbf{P}^{2} /\left(2 m_{2}\right)+V_{0} & \text { elsewhere }\end{cases}
$$

where $\mathbf{r}$ and $\mathbf{P}$ are the electron coordinate and momentum. $m_{1}, m_{2}$ and $V_{0}$ are the carrier band effective mass in the well material and the barrier material and the carrier-confining potential, respactively. $y_{1}(x)$ and $y_{2}(x)$ describe the upper and bottom boundaries of ridge quantum wire, Fig. 1 . The following coordinate transformation transforms the V-shaped boundaries into planar ones

$$
\begin{gathered}
x^{\prime}=x \\
y^{\prime}=-y-b \tan (\theta) \ln [\cosh (x / b)] . \\
z^{\prime}=z
\end{gathered}
$$

where $b$ is the bend width at the top of the ridge and the angle $\theta$ is such that $\left(180^{\circ}-2 \theta\right)$ is the actual angle between the facets of the ridge. The transformed Schrödinger equation becomes considerably more complicated as it now contains all the information about the lateral confinement.

$$
\begin{aligned}
& -\frac{\hbar^{2}}{2 m^{*}}\left[\frac{\partial^{2} \psi}{\partial x^{\prime 2}}+\left(1+\tan ^{2}(\theta) \tanh ^{2}\left(x^{\prime} / b\right)\right) \frac{\partial^{2} \psi}{\partial y^{\prime 2}}\right. \\
& \left.-\frac{\partial}{\partial y^{\prime}}\left(\tan (\theta) \tanh \left(x^{\prime} / b\right) \frac{\partial \psi}{\partial x^{\prime}}\right)-\frac{\partial}{\partial x^{\prime}}\left(\tan (\theta) \tanh \left(x^{\prime} / b\right) \frac{\partial \psi}{\partial y^{\prime}}\right)\right] \\
& +V\left(y^{\prime}\right) \psi=E \psi .
\end{aligned}
$$


In the transformed coordinates the potential barrier becomes a function of $y^{\prime}$ only, such as:

$$
V\left(y^{\prime}\right)= \begin{cases}0 & \text { if }\left|\mathrm{y}^{\prime}\right|<\mathrm{L} / 2 \\ V_{0} & \text { if }\left|\mathrm{y}^{\prime}\right|>\mathrm{L} / 2\end{cases}
$$

and

$$
\begin{aligned}
E & =\frac{\int \psi^{*}(\mathbf{r}) H(\mathbf{r}) \psi(\mathbf{r}) d V}{\int \psi(\mathbf{r}) \psi(\mathbf{r}) d V} \\
& =\frac{\int \psi^{*}\left(\mathbf{r}^{\prime}\right)\left|J\left(\mathbf{r}^{\prime}\right)\right| H\left(\mathbf{r}^{\prime}\right) \psi\left(\mathbf{r}^{\prime}\right) d V^{\prime}}{\int \psi^{*}\left(\mathbf{r}^{\prime}\right)\left|J\left(\mathbf{r}^{\prime}\right)\right| \psi\left(\mathbf{r}^{\prime}\right) d V^{\prime}}
\end{aligned}
$$

where $L$ and $J\left(\mathbf{r}^{\prime}\right)$ are the channel width along the $y$ direction and the Jacobian determinant. In the new coordinate space, the V-groove quantum wire becomes a quantum well with a confining potential in the $\mathrm{x}$ direction. All mixed terms of the Eq.(3) is replaced by an effective lateral potential [15]:

$$
V_{e f f}\left(x^{\prime}\right)=V_{x^{\prime}} \tan (\theta) \tanh ^{2}\left(x^{\prime} / b\right),
$$

where $V_{x^{\prime}}$ is an angle-independent barrier factor. This form of the lateral potential is proportional to the angle between the facets of the ridge, which allows the decoupling of the two-dimensional Schrödinger equation into two readily solved one-dimensional Schrödinger equations. These equations were analytically solved and the wave functions and energy levels for the carrier were obtained [16]. For ground state, the wave functions of carrier are given as:

$$
\begin{aligned}
\psi\left(x^{\prime}, y^{\prime}\right) & =\phi\left(x^{\prime}\right) \phi\left(y^{\prime}\right) \\
& =\operatorname{sech}\left(x^{\prime} / b\right) \exp \left(k y^{\prime}\right) \quad y^{\prime}<-L / 2 \\
& =\operatorname{sech}\left(x^{\prime} / b\right) \cos \left(q y^{\prime}\right) \quad\left|y^{\prime}\right|<L / 2 \\
& =\operatorname{sech}\left(x^{\prime} / b\right) \exp \left(-k y^{\prime}\right) \quad L / 2<y \\
k^{2}= & \frac{2 m_{2}}{\hbar^{2}}\left(V_{0}-E\right), \quad q^{2}=\frac{2 m_{1} E}{\hbar^{2}} .
\end{aligned}
$$

The Hamiltonian for shallow donor impurity states can be written as

$H_{i m}(\mathbf{r})=-\frac{e^{2}}{\varepsilon\left|\mathbf{r}-\mathbf{r}_{i}\right|}+ \begin{cases}\mathbf{P}^{2} /\left(2 m_{1}\right) & \mathrm{y}_{2}(\mathrm{x})<\mathrm{y}<\mathrm{y}_{1}(\mathrm{x}) \\ \mathbf{P}^{2} /\left(2 m_{2}\right)+V_{0} & \text { elsewhere }\end{cases}$

where $\varepsilon$ and $\mathbf{r}_{i}$ are the dielectric constant and the impurity position, respectively. The impurity binding energy is calculated by a traditional variational method, due to no exact solutions to the impurity states in quantum wire. The following trial wave function in transformed coordinate is adopted for ground impurity state in ridge quantum wire:

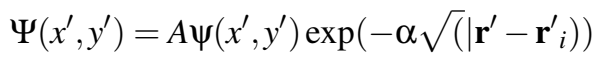

where $\psi\left(x^{\prime}, y^{\prime}\right)$ is the ground state wave function without impurity, Eq.(7). The $\alpha$ is the variational parameter, and will be determined by minimizing the expectation value of the Hamiltonian $E=\left\langle\Psi\left|H_{i m}\right| \Psi\right\rangle$. The impurity binding energy, which is defined as the difference between the lowest value of the energy for the system without the impurity and the minimized value of $E$, is obtained numerically.

$$
E_{b}=E_{0}-\min _{\alpha} \frac{\left\langle\Psi\left(x^{\prime}, y^{\prime}\right)\left|J\left(\mathbf{r}^{\prime}\right)\right| H_{i m} \Psi\left(x^{\prime}, y^{\prime}\right)\right\rangle}{\left\langle\Psi\left(x^{\prime}, y^{\prime}\right)\left|J\left(\mathbf{r}^{\prime}\right)\right| \Psi\left(x^{\prime}, y^{\prime}\right)\right\rangle}
$$

all calculations are carried out in transformed coordinates.

\section{RESULTS AND DISCUSSION}

The impurity states are an important factor to affect the electric-transportation and optical properties in lowdimensional semiconductor structures. The binding energies and wave function are calculated for $G a A s / G a_{0.7} A l_{0.3} A s \mathrm{~V}$ grooved quantum wire. The material parameters used in calculations are: $m_{e}^{*}=0.067$, dielectric constant $\varepsilon=13.18 \varepsilon_{0}$, the barrier potential $V_{0}=264 \mathrm{meV}$ and the barrier factor $V_{X}=42.4 \mathrm{meV}$ for the conduction band [17]. The angle is $\theta=54.75^{\circ}$ such that $180^{\circ}-2 \theta$ corresponds to the measured angle between the two facets of the wire $[15,16]$.

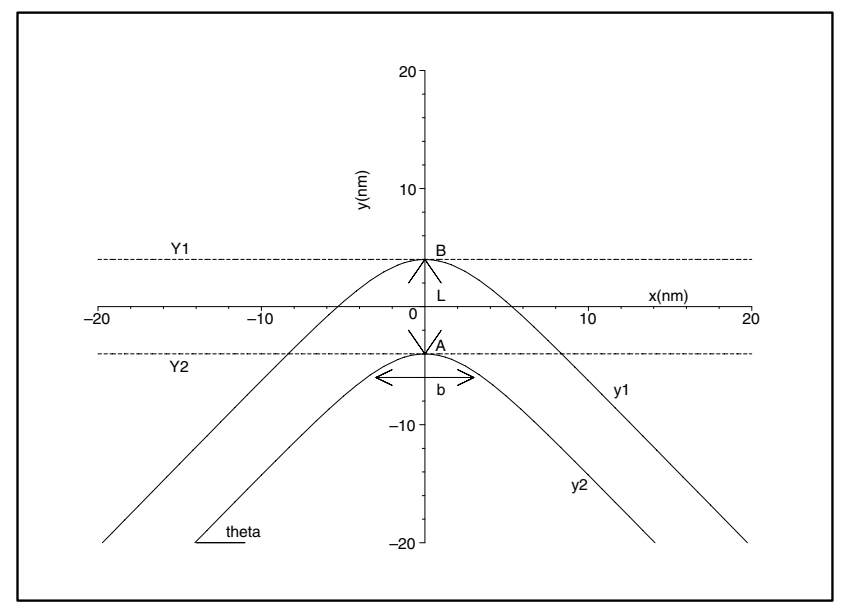

FIG. 1: A schematic representation of ridge quantum wires. Solid curves are original coordinates and dashed lines are the transformed interfaces.

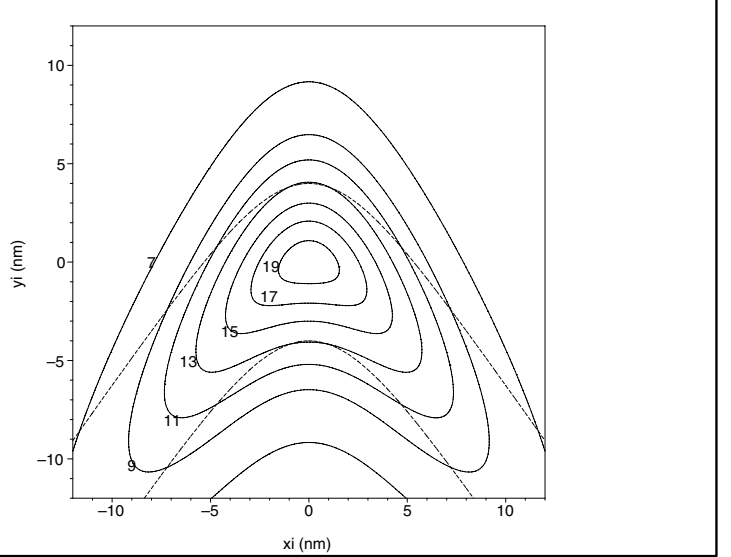

FIG. 2: Contour plots of the donor binding energy for $L=8 \mathrm{~nm}$, $b=4 \mathrm{~nm}, \theta=54.75^{\circ}$. The contour plots show curves with the same binding energy. Dashed lines show the position of the interfaces.

The variational parameter which minimizes the expectation value of the Hamiltonian of the system was calculated for different positions of the impurity inside the wire. The donor binding energies as a function of the impurity position $x_{i}$ and $y_{i}$ are shown in Fig. 2 for a V-groove quantum wire 
with $L=8 \mathrm{~nm}$ and $b=4 \mathrm{~nm}$. As it is seen, the binding energy is maximum for a donor located at the center of the Vgroove quantum wire and decreases as the donor position is moved away from the center. A larger decrease is also observed for donors located in the barrier region. The quantitative and qualitative behaviours of the binding energies are in close agreement with the calculations of Weber and de Paula [17] for similar structures.

The wave functions for two impurity positions in the quantum wires are shown in Figs.3-4. For an impurity located at the center of the wire, the wave function is localized in the central region Fig. 3, and for impurities located other positions, the wave functions become strongly deformed Fig. 4. Penetration of the impurity wave functions into barrier regions are also seen in Figs.3-4. These figures are also in good agreement with the numerical analysis by Weber [17].

(a)

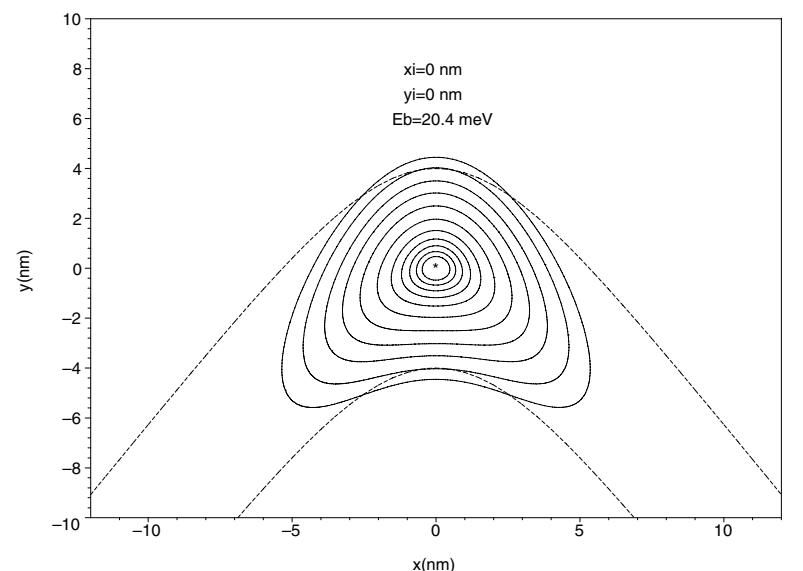

(b)

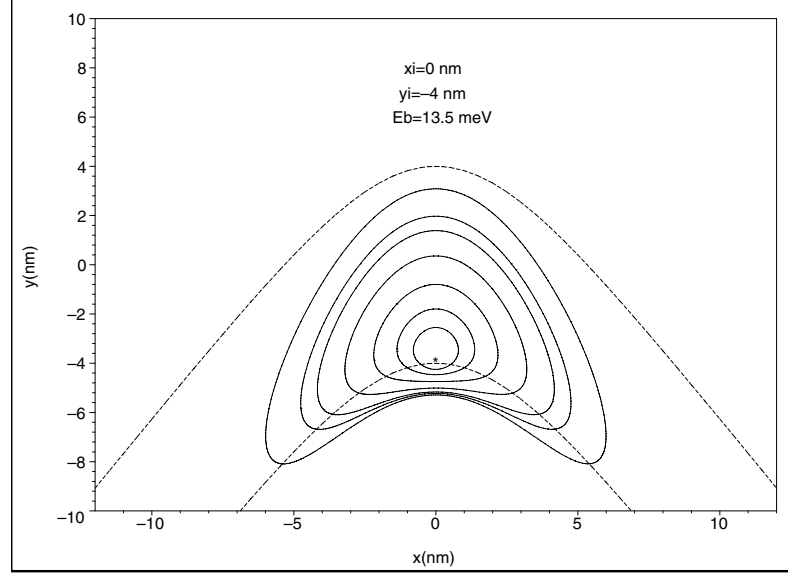

FIG. 3: Contour plots of the impurity wave function in the V-groove quantum wire with $L=8 \mathrm{~nm}, b=4 \mathrm{~nm}$ and $\theta=54.75^{\circ}$ for two different impurity positions.

The variations of impurity binding energy versus dimension of the wire are shown in Fig. 5. Where o is the center of quantum wire and $\mathrm{A}$ and $\mathrm{B}$ are the cross points of the bottom and upper $\mathrm{V}$-shaped boundaries with $y$-axis, Fig. 1. As it is

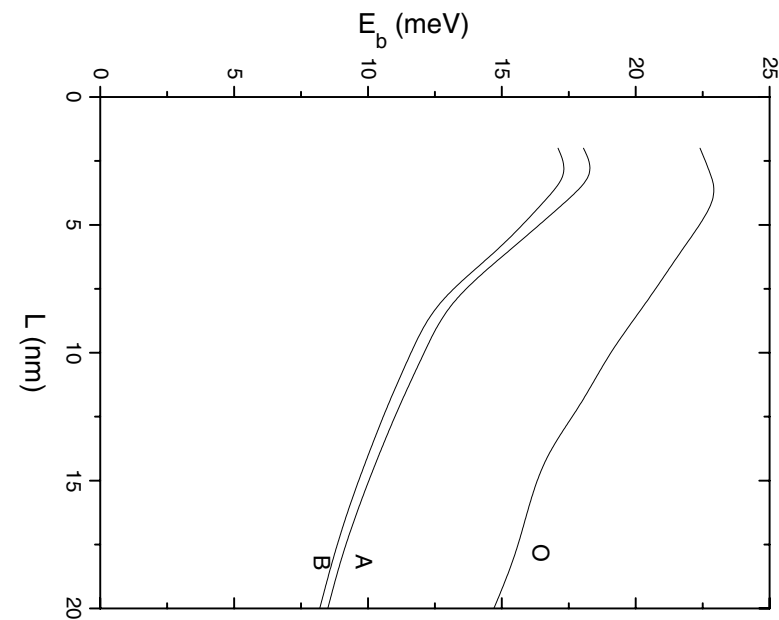

FIG. 4: Variations in impurity binding energy versus the dimension of ridge quantum wires for three different impurity positions.

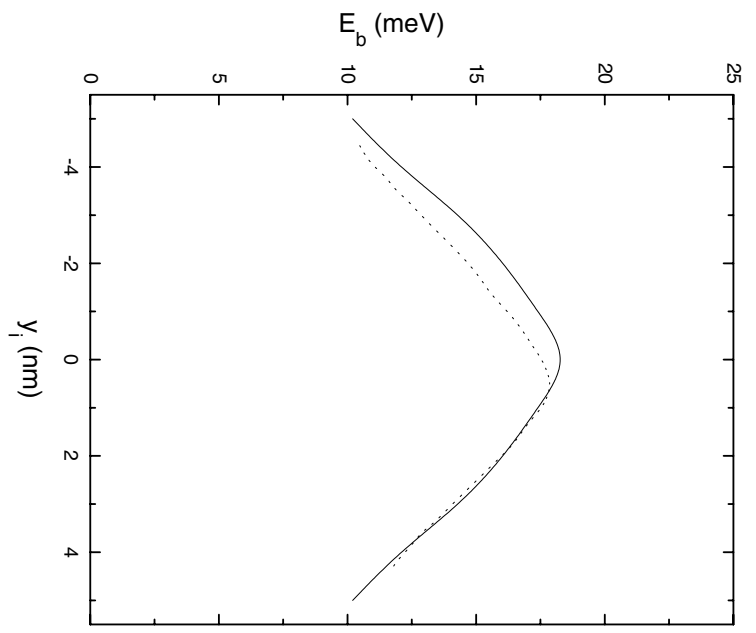

FIG. 5: Variations in impurity binding energy as a function of the impurity position along the $y$ axis $(x=0)$ for $L=8.8 \mathrm{~nm}$ (solid curve). The dotted line corresponds to the experimental work done by Vouilloz et.al.

seen, when the dimension of ridge quantum wire decreases, the impurity binding energy at three points increases at first, to a maximum value, and then decreases. These behaviours are similar to the impurity state in traditional quantum wires with finite confining potential [18]. This phenomenon is related to the change of the electron confinement in ridge quantum wires. When the dimension of V-groove quantum wire decreases, the confinement of electrons is strengthened, and therefore the impurity binding energy increases. Because the practical V-groove quantum wires with finite confining potential are considered here, and when the dimension of V-groove quantum wires is reduced to a small limited value, most of the electronic wave functions begin to leak out of the well region, which results in a maximum impurity binding energy at a small $L$ value. It is also interesting to note that the impurity binding energy at the center is larger than that two boundary 
points. The impurity binding energy at the bottom boundary point is larger than at the upper boundary point, which indicates an asymmetrical distribution of impurity binding energy along the direction normal to the boundaries. This difference is different from the impurity state behaviour in traditional quantum wire structures $[10,18]$.

In order to understand clearly the asymmetrical distribution of impurity binding energy in V-groove quantum wires, the dependence of binding energy on the impurity position along the $y$-axis for $L=8.8 \mathrm{~nm}$ and $\theta=54.75^{\circ}$ is shown in Fig.6, solid curve. For comparison the experimental work done by Vouilloz et al [19] for a quantum wire with $L=8.8 \mathrm{~nm}$ and different curvatures is also shown in this figure, dashed curve. As is seen, the calculated results are close to the experimental value. The asymmetrical distribution of impurity binding energy along the $y$ direction can be clearly seen, and the weighted center of the binding energy distribution in the $y$ di- rection moves to the convex side of quantum wire.

\section{CONCLUSION}

We calculated shallow impurity binding energies in Vgroove quantum wires using an effective potential method for the calculation of the electron and hole ground states. It was found that the impurity state behaviour in ridge quantum wire in a sense is similar to that in traditional quantum wires. However, the distribution of impurity binding energy along the direction normal to the V-shaped boundaries is asymmetrical, and the impurity position corresponding to the maximum binding energy deviates from the center, due to the asymmetrical confining potential produced by the V-shaped boundaries in the $y$ direction.
[1] J. B. Xia and K. W. Cheah, Phys. Rev. B 55, 1596 (1997).

[2] R. Cingolani, F. Sogawa, Y. Arakawa, R. Rinaldi, M. DeVittorio, A. Passaseo, A. Taurino, M. Catalano, and L. Vasanelli, Phys. Rev. B 58, 1962 (1998).

[3] E. Martinet, F. Reinhardt, A. Gustafsson, G. Biasol, and E. Kapon, Appl. Phys. Lett. 72, 701 (1998).

[4] M. Kappelt, M.Grundmann, A. Krost, V. Turck, and D. Bimberg, Appl. Phys. Lett. 68, 3596 (1996).

[5] G. Biasiol, E. Kapon, Y. Ducommun, and A. Gustafson, Phys. Rev. B 57, R9416 (1998).

[6] S. Pescetelli, A. Di Carlo, and P. Lugli, Phys. Rev. B 56, R1668 (1997).

[7] A. Sa'ar, S. Calderon, A. Givant, O. Ben-Shalom, E. Kapon, and C. Caneau, Phys. Rev. B 54, 2675 (1996).

[8] C. Ammann, M. A. Dupertius, U. Bockelmann, and B. Deveaud, Phys. Rev. B 55, 2420 (1997).

[9] J. W. Brown and H. N. Spector, J. Appl. Phys. 59, 1179 (1986).
[10] G. W. Bryant, Phys. Rev. B 29, 6632 (1984); 31, 7812 (1985).

[11] R. Rinaldi et al, Phys. Rev. Lett. 73, 2899 (1994).

[12] J. F. Ryan, A. C. Maciel, C. Kiener, L. Rota, K. Turner, J. M. Freyland, U. Marti, D. Martin, F. Morier-Gemoud, and F. K. Reinhart, Phys. Rev.B 53, R4225 (1996).

[13] K.Chang and J. B. Xia, Phys. Rev. B58, 2031 (1998).

[14] T. Ionshita and H. Sakaki, J. Appl. Phys. 79, 269 (1996).

[15] G. Creci and G. Weber, Semicond. Sci. Technol. 14, 690 (1999).

[16] E. Sadeghi, R. Khordad, Phys. Stat. Sol. (b) 242, 1628 (2005).

[17] G. Weber and Ana M. de Paula, Phys. Rev. B 63, 113307 (2001).

[18] Z. Y. Deng, T. R. Lai, J. K. Guo, and S. W. Gu, J. Appl. Phys. 75, 7389 (1994).

[19] F. Vouilloz, D. Y. Oberli, M. A. Dupertuis, A. Gustafsson, F. Reinhardt, and E. Kapon, Phys. Rev. Lett. 78, 1580 (1997); Phys. Rev. B57, 12378 (1998). 